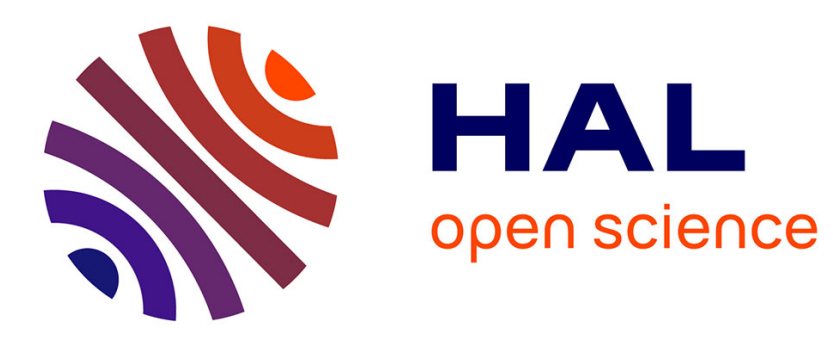

\title{
Nonlinear multiple scattering of acoustic waves by a layer of bubbles
}

Olivier Lombard, Christophe Barrière, Valentin Leroy

\section{To cite this version:}

Olivier Lombard, Christophe Barrière, Valentin Leroy. Nonlinear multiple scattering of acoustic waves by a layer of bubbles. EPL - Europhysics Letters, 2015, 10.1209/0295-5075/112/24002 . hal01527080

\section{HAL Id: hal-01527080 \\ https://hal.science/hal-01527080}

Submitted on 23 May 2017

HAL is a multi-disciplinary open access archive for the deposit and dissemination of scientific research documents, whether they are published or not. The documents may come from teaching and research institutions in France or abroad, or from public or private research centers.
L'archive ouverte pluridisciplinaire HAL, est destinée au dépôt et à la diffusion de documents scientifiques de niveau recherche, publiés ou non, émanant des établissements d'enseignement et de recherche français ou étrangers, des laboratoires publics ou privés. 


\title{
Nonlinear multiple scattering of acoustic waves by a layer of bub- bles
}

\author{
O. LOMBARD ${ }^{1}$, C. BARRIÈRE ${ }^{2}$, V. LEROY ${ }^{1}$ \\ 1 Laboratoire Matière et Systèmes Complexes (MSC) - Université Paris-Diderot, CNRS UMR 7057, Paris, France. \\ 2 Institut Langevin, ESPCI ParisTech, CNRS (UMR 7587), PSL Research University, Paris, France.
}

PACS 43.25.+y - Nonlinear acousticsVibrations and mechanical waves

PACS 46.40.Cd - Mechanical wave propagation (including diffraction, scattering, and dispersion)

\begin{abstract}
We present a theoretical and experimental study of the acoustic second-harmonic generation by a single layer of bubbles. This simple system allows us to investigate the subtle interplay between nonlinear effects and multiple scattering. A perturbative model is shown to give an excellent agreement with the experimental measurements, and we demonstrate the existence of an optimal concentration of bubbles, for which the harmonic generation is maximum. The potential of bubble screens as efficient subwavelength acoustic nonlinear sources is discussed.
\end{abstract}

Introduction. - Wave transport in a multiple scattering environment has been a subject of intense research, demonstrating a large variety of behaviors, from the dispersive propagation of a coherent wave $[1,2]$ to the existence of a diffusive regime, sometimes leading to localization $[3,4]$. On the other hand, the nonlinear propagation of waves also comes with many intriguing phenomena, such as self-induced transparency [5] or secondharmonic generation [6], for instance. The question arises of how waves propagate when both strong multiple scattering and nonlinearities are present. For mechanical waves, this question has been addressed in granular media [7,8], with the complication that both scattering and nonlinearities are strongly dependent on the contact between the grains. Gas bubbles appear as perfect candidates for looking at nonlinear acoustic propagation in a multiple scattering regime: they are efficient acoustic scatterers, as well as strong nonlinear sources. Bubbly liquids have already been shown to exhibit substantial acoustic nonlinearities [9-12]. But an important limitation of the previous studies was the lack of quantitative comparison between the theoretical predictions and the experimental measurements, often due to a weak knowledge of the structure of the bubbly liquids used for the experiments.

In this Letter, we use stable and well-characterized bubbly media to carefully study the interplay between nonlinearities and multiple scattering. Our experimental system is a single layer of bubbles, organized on a square lattice (see inset of Fig. 1). We focus on a particular nonlin- ear mechanism: harmonic generation, i.e. how a pressure wave at frequency $f$ generates, due to the presence of bubbles, a wave at frequency $2 f$.

Theory. - We limit ourselves to the long wavelength regime, which means that the wavelength is much larger than the typical distances involved in the system, namely the radius $R$ of the bubbles, and the distance $d$ between two neighboring bubbles.

Linear regime. Excited by a monochromatic pressure with complex amplitude $P, P \exp [-i \omega t]$, a bubble oscillates and generates at distance $r$ a spherical pressure field $p(r, t)=\ddot{V}(t-r / c) /(4 \pi r)$, where $V(t)$ is the instant bubble volume and $c$ the sound speed in the liquid. With a linear development of Rayleigh-Plesset equation [13], this pressure field can be written $p(r, t)=f_{s} P \exp [i \omega(r / c-t)] / r$, where the scattering function

$$
f_{s}(\omega)=\frac{R}{\left(\frac{\omega_{0}}{\omega}\right)^{2}-1-i(k R+\delta)}
$$

has been introduced. In Eq. (1), $\omega_{0}$ is the resonance angular frequency of the bubble and $k R+\delta$ the total damping constant ( $k=\omega / c$ is the wavenumber in the liquid). The damping of the bubble is due to radiative losses (the $k R$ term) and dissipation (the $\delta$ term), the latter being divided into a viscous and a thermal contribution.

As they are strong scatterers, bubbles couple efficiently one to each other: if several bubbles are present in a liquid, the total pressure field experienced by one bubble is 


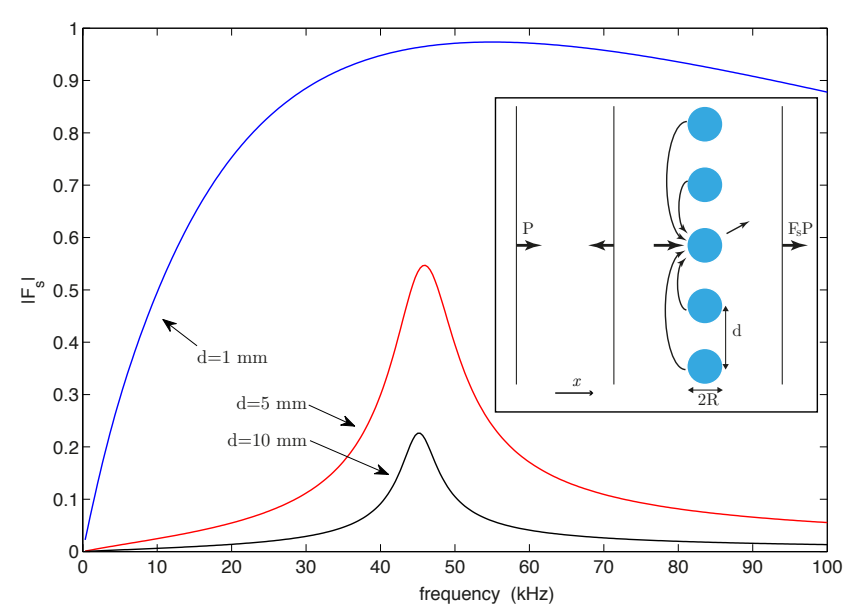

Fig. 1: Modulus of the linear response function $F_{s}$ for a single layer of air bubbles in water, as a function of frequency. Here the radius of the bubbles is $R=70 \mu \mathrm{m}$ and three different lattice constants are considered: $d=1,5$ and $10 \mathrm{~mm}$. The inset is a schematic view of the scattering process in the layer: each bubble generates a spherical wave in response to the total exciting pressure wave, which is the sum on the incident wave $P$ and the waves scattered by all the other bubbles. The contributions of all these spherical fields make, in the far field, two plane waves $F_{s} P$, in the forward and backward directions.

not reduced to the incident field; the contribution of the other bubbles must be taken into account. An insightful situation is given by the particular case of a single layer of identical bubbles organized on a square lattice. When the layer is excited by a normal incident plane wave $P \exp [i \omega(x / c-t)]$, all the bubbles experience the same total pressure field (due to the translation invariance of the system), which can be evaluated on a particular bubble, labelled 0 (see figure 1) [14],

$$
P_{t o t}=P+\sum_{|p|>0} \sum_{|q|>0} P_{t o t} f_{s} \frac{\mathrm{e}^{i \frac{\omega d}{c} \sqrt{p^{2}+q^{2}}}}{d \sqrt{p^{2}+q^{2}}} .
$$

It has been shown that the discrete sum could be approximated by a continuous integral:

$$
\begin{aligned}
P_{t o t} & \approx P+P_{t o t} \int_{b}^{+\infty} f_{s} \frac{e^{i \frac{\omega r}{c}}}{r} \frac{1}{d^{2}} d S \\
& =P\left(1-i \frac{2 \pi c}{\omega d^{2}} f_{s} e^{i \frac{\omega b}{c}}\right)^{-1}
\end{aligned}
$$

where $b=d / \sqrt{\pi}$ is a cut-off distance taking into account the positional correlations induced by the square lattice. Then it can be shown that, at distance $x$ from the layer, the contributions of all the bubbles sum up into a plane wave $P F_{s} \exp [i \omega(x / c-t)]$ with a response function $F_{s}$ given by

$$
F_{s}(\omega)=\frac{i K R}{\left(\frac{\omega_{0}}{\omega}\right)^{2}-1+2 \pi \frac{R b}{d^{2}}-i(K R+\delta)},
$$

where $K=\lambda / d^{2}(\lambda=2 \pi c / \omega$ is the wavelength $)$. Eq. (4) takes, as Eq. (1), the form of an harmonic oscillator response function. Figure 1 shows the magnitude of $F_{s}$ as a function of frequency, for layers of different lattice constants. The maximum of $\left|F_{s}\right|$ is reached for a frequency that corresponds to the resonance frequency of the individual bubbles $\left(\omega_{0} / 2 \pi=45 \mathrm{kHz}\right.$ for a $R=70 \mu \mathrm{m}$ air bubble in water) when the lattice constant $d$ is large compared to the radius of the bubbles (small concentration). For small values of $d / R$ (high concentration), the peak of the response is shifted to a higher frequency. However, the main effect of the coupling is not on the position of the peak but on its width and amplitude. This effect is piloted by the $K R$ term that appears in Eq. (4), which makes the peak broader and higher when $d$ decreases. In the linear regime, the more bubbles in the layer (i.e. small $d$ ), the stronger the response.

Nonlinear regime. In order to model nonlinear effects, the Rayleigh-Plesset equation is developed to the second order. When a bubble is excited by two frequencies, $P_{1} \exp \left[-i \omega_{1} t\right]+P_{2} \exp \left[-i \omega_{2} t\right]$, its oscillation has a non linear component, at frequency $\omega_{1}+\omega_{2}$. As a consequence, it generates at distance $r$ a spherical pressure field $p^{(2)}(r, t)=2 f_{s}^{(2)} P_{1} P_{2} \exp \left[i\left(\omega_{1}+\omega_{2}\right)(r / c-t)\right] / r$ with a non linear scattering function calculated by a perturbative method:

$$
f_{s}^{(2)}\left(\omega_{1}, \omega_{2}\right)=\frac{f_{s}\left(\omega_{1}\right) f_{s}\left(\omega_{2}\right) f_{s}\left(\omega_{1}+\omega_{2}\right)}{6 \kappa P_{g} R^{2}} A_{12},
$$

with $A_{12}=\left(\omega_{0} / \omega_{1}\right)^{2}+\left(\omega_{0} / \omega_{2}\right)^{2}+\omega_{0}^{2} /\left(\omega_{1} \omega_{2}\right)-3(\kappa+$ 1) $\omega_{0}^{4} /\left(\omega_{1} \omega_{2}\right)^{2}-3 i\left[\left(\omega_{0} / \omega_{2}\right)^{2}+\left(\omega_{0} / \omega_{1}\right)^{2}+2 \omega_{0}^{2} /\left(\omega_{1} \omega_{2}\right)\right] \delta\left(\omega_{1}+\right.$ $\left.\omega_{2}\right)$, and where $P_{g}$ is the pressure of the gas in the bubble (at rest) and $\kappa$ its polytropic exponent.

As in the linear case, going from the single bubble to the layer of bubbles imposes to take mutliple scattering into account. As depicted in the inset of Fig. 2, two types of processes lead to the nonlinear response of the layer. In total, one finds that when excited by two plane waves $P_{1} \exp \left[i \omega_{1}(x / c-t)\right]+P_{2} \exp \left[i \omega_{2}(x / c-t)\right]$, a layer of bubbles emits a nonlinear plane wave $2 F_{s}^{(2)} P_{1} P_{2} \exp \left[i\left(\omega_{1}+\right.\right.$ $\left.\left.\omega_{2}\right)(x / c-t)\right]$ with a nonlinear response function $F_{s}^{(2)}$ given by :

$$
F_{s}^{(2)}\left(\omega_{1}, \omega_{2}\right)=-\frac{F_{s}\left(\omega_{1}\right) F_{s}\left(\omega_{2}\right) F_{s}\left(\omega_{1}+\omega_{2}\right)}{6 \kappa P_{g}} \frac{d^{4} A_{12}}{R^{2} \lambda_{1} \lambda_{2}}
$$

where $\lambda_{i}$ is the wavelength in the liquid at $\omega_{i}$. A simple case of particular interest is when a single frequency $\omega$ is exciting the layer: the incident field is $P \exp [i \omega(x / c-$ $t)]$. The nonlinear process then corresponds to harmonic generation: the layer emits a plane wave

$$
p(x, t)=F_{s}^{(2)} P^{2} \exp [i 2 \omega(x / c-t)],
$$

with 


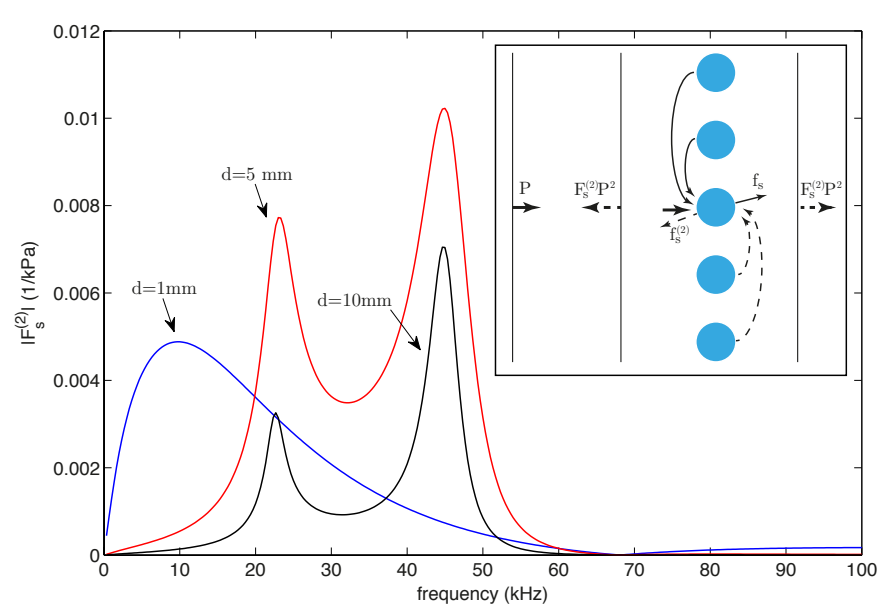

Fig. 2: Modulus of the nonlinear response function $F_{s}^{(2)}$ in the case of harmonic generation, for the same bubble layers as in figure 1 . The inset shows that the harmonic wave generated by a single bubble is due to its nonlinear response to the total linear field it experiences, as well as to its linear response to the nonlinear field generated by the other bubbles. As in the linear case, the sum of all the contributions makes, in the far field, a plane wave $F_{s}^{(2)} P^{2}$.

$$
F_{s}^{(2)}(\omega)=-\frac{\left[F_{s}(\omega)\right]^{2} F_{s}(2 \omega)}{2 \kappa P_{g}(K R)^{2}}\left[\frac{\omega_{0}^{2}}{\omega^{2}}-(\kappa+1) \frac{\omega_{0}^{4}}{\omega^{4}}\right],
$$

where the imaginary part of $A_{12}$ has been neglected. The result of this equation is plotted in Fig. 2 for the same layers of bubbles considered in the linear case. For the two dilute layers $(d=5$ and $10 \mathrm{~mm})$ two peaks are apparent at $\omega_{0} / 2$ and $\omega_{0}$, which is expected because Eq. (8) shows that the harmonic response depends on both $F_{s}(2 \omega)$ (maximum for $\omega=\omega_{0} / 2$ ) and $F_{s}(\omega)$ (maximum for $\omega=\omega_{0}$ ). For $d=1 \mathrm{~mm}$, however, the nonlinear response is different. A striking difference between the linear and the nonlinear cases is that the strongest response is not given by small values of $d$. As obvious in Fig. 2, at the resonance frequency, the nonlinear response is not favored by the concentrated $d=1 \mathrm{~mm}$ case; it is higher for the intermediate $d=5 \mathrm{~mm}$ case. It means that, contrary to the linear case, the nonlinear response does not depend monotonously on the concentration of bubbles in the layer: there is an optimal concentration.

The optimal distance between bubbles can be found by analysing Eq. (8). At resonance, $F_{s}\left(\omega_{0}\right)=K R /(K R+\delta)$ and $F_{s}\left(2 \omega_{0}\right) \approx-i K R / 3$ (see equation $\left.(4)\right)$, so equation (8) reduces to

$$
F_{s}^{(2)}\left(\omega_{0}\right)=\frac{i}{3 P_{g}} \times \frac{K R}{(K R+\delta)^{2}},
$$

which reaches a maximum value

$$
F_{s, \max }^{(2)} \simeq \frac{i}{12 P_{g} \delta} \text { for } K R=\delta \rightarrow d=\sqrt{\frac{\lambda R}{\delta}}
$$

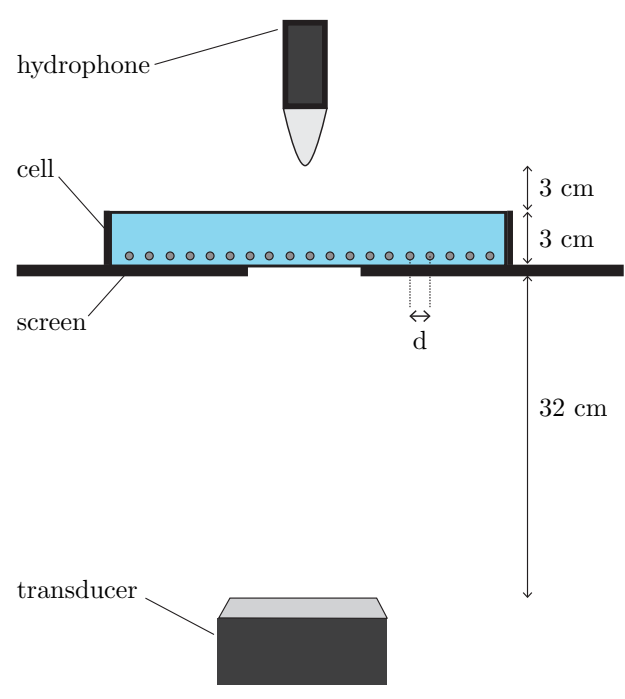

Fig. 3: Sketch of the experimental setup. A transducer emits an acoustic signal which is transmitted through the layer of bubbles, and measured by an hydrophone. A thin wall cell filled with a yield-stress fluid is used to trap the bubbles. The cell is placed close to a screen with a $5 \mathrm{~cm}$-diameter aperture to limit spurious signals coming from the edges.

For $R=70 \mu \mathrm{m}$ air bubbles in water, at resonance one has $\lambda=3.3 \mathrm{~cm}$ and $\delta=0.08$ (mainly due to thermal losses), Eq. (10) predicts a maximum of $0.01 \mathrm{kPa}^{-1}$ for $d=5.4 \mathrm{~mm}$, in good agreement with figure 2 .

The physical origin of this optimal concentration of bubbles comes from the interplay between nonlinearity and multiple scattering. On one hand, generation of harmonic is favored by a large number of nonlinear sources, i.e. a small $d$. On the other hand, the total pressure field on each bubble of the layer decreases when $d$ decreases, making the nonlinear sources less efficient. This effect can be appreciated by looking closer to equation (3), which shows how the incident field $P$ is modified by the presence of other bubbles. At resonance $f_{s}=i R /(k R+\delta)$, and the total pressure field is

$$
P_{\mathrm{tot}} \simeq P \frac{d^{2}(k R+\delta)}{d^{2}(k R+\delta)+\lambda R},
$$

which decreases for decreasing values of $d$. For the optimal concentration, for instance, the total pressure is only half of the incoming pressure. In summary, many bubbles make many nonlinear sources, but too many bubbles make the sources less efficient, which explain the existence of an optimal lattice constant $d$.

Experiments. - Controlled and stable samples were obtained by injecting bubbles in a yield-stress fluid [14]. In this kind of fluid, one can move a capillary to inject bubbles at any desired positions, the bubbles being trapped in the fluid if the yield stress is large enough to compensate the buoyant force. A single layer of bubbles was injected in a thin-wall cell, which was then placed in a large wa- 

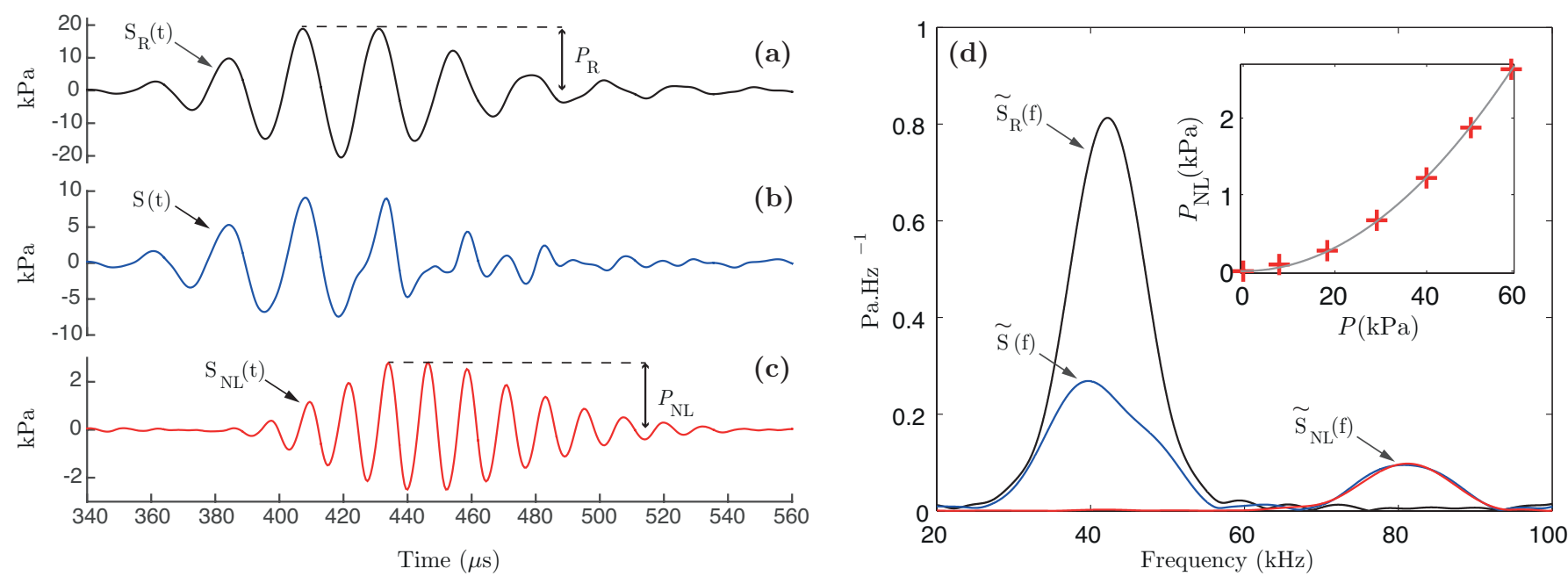

Fig. 4: Examples of acquired signals for a $P=60 \mathrm{kPa}$ incident amplitude: (a) reference signal, (b) signal through the layer of bubbles (here $R=70 \mu \mathrm{m}$ and $d=3.5 \mathrm{~mm}$ ), (c) nonlinear component obtained by pulse inversion (see text). (d): Amplitude of the Fourier transforms of the different signal, and amplitude of the nonlinear signal as a function of the incoming pressure $P$ (inset).

ter tank for measurement of the acoustic transmission, as schematized in figure 3. Because the resonance frequency of the bubbles was particularly low, special care was given to diffraction. Indeed, for the smallest bubbles we were able to inject $(R=70 \mu \mathrm{m})$, the resonance frequency was of the order of $40 \mathrm{kHz}$, which is lower than the range of frequencies used for conventional ultrasonic techniques. At this frequency, the wavelength in water is $3.75 \mathrm{~cm}$. We used a large transducer (SY-80, Hz Sonic Ltd, $15 \mathrm{~cm}$ in diameter) to limit beam spreading, and a screen with a $5 \mathrm{~cm}$-diameter aperture to make sure that no signal was leaking around the sample. To accurately separate the direct signal transmitted by the bubble screen from the multiple echoes in the tank, we used a short pulse signal rather than a continuous emission. The transducer was excited by a gaussian voltage pulse $V \cos \left(2 \pi f_{c} t\right) \exp \left[-(2 \pi \sigma t)^{2} / 2\right]$ with a central frequency $f_{c}=42 \mathrm{kHz}$, a frequency width $\sigma=5 \mathrm{kHz}$, and an amplitude $V$ up to $150 \mathrm{~V}$, provided by a power amplifier. To determine the pressure excitation undergone by the bubbles, the pressure level was measured close to the aperture of the screen, in the absence of the bubble sample. A linear dependence was found between the voltage and the pressure, with a maximum pressure of $P=60 \mathrm{kPa}$.

Typical measurements are shown in figure 4 . We note $S_{R}(t)$ the reference signal, acquired $6 \mathrm{~cm}$ away from the screen (see Fig. 4a). When a layer of bubbles is placed in the acoustic field, the amplitude is reduced and nonlinear features appear in the last part of the signal (see Fig. 4b). To better appreciate the nonlinear component of the signal, we used a pulse inversion technique: transmitted signals with opposite voltages were acquired and the average signal $S_{N L}(t)$ was calculated, thus eliminating the linear contributions. As can be seen in Fig. 4c, there is indeed harmonic generation by the layer of bub- bles: the frequency of the nonlinear pulse is twice that of the incoming signal. Note that this pulse arrives with a substantial delay. The amplitude of the nonlinear response, $P_{N L}$, was checked to vary quadratically with the applied pressure, as shown in the inset of Fig. 4d.

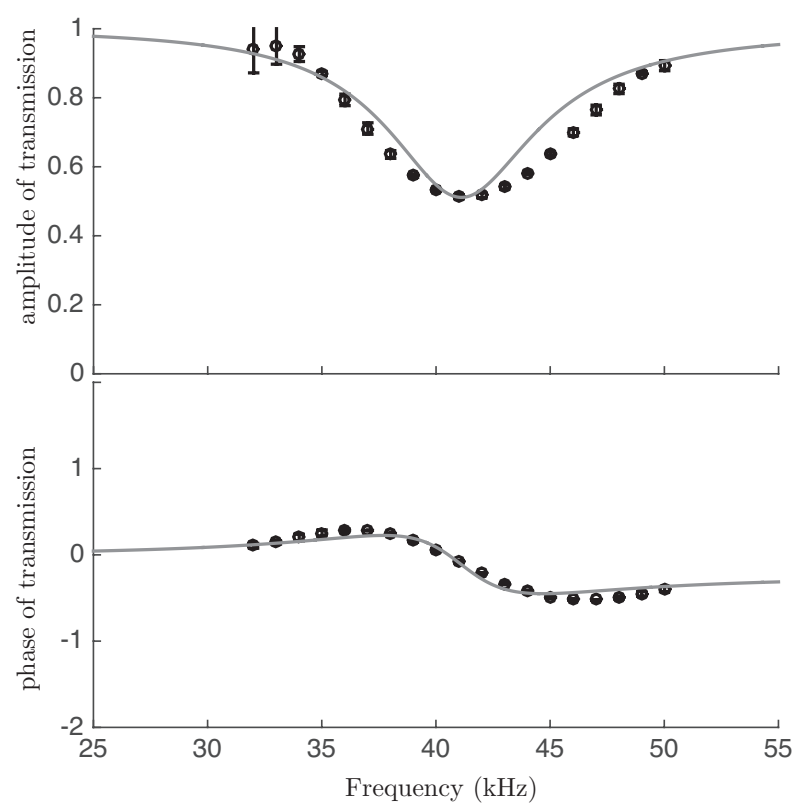

Fig. 5: Amplitude and phase of the linear transmission as a function of frequency, for a layer of bubbles with $R=70 \mu \mathrm{m}$ and $d=5 \mathrm{~mm}$. Symbols are the experimental measurements, solid lines the theoretical prediction $\left(1+F_{s}\right)$.

Linear results. Fig. 5 compares the amplitude and phase of the measured linear transmission with the theoretical prediction $1+F_{s}$. The good agreement indicates that the sources of dissipation for the oscillations of the 


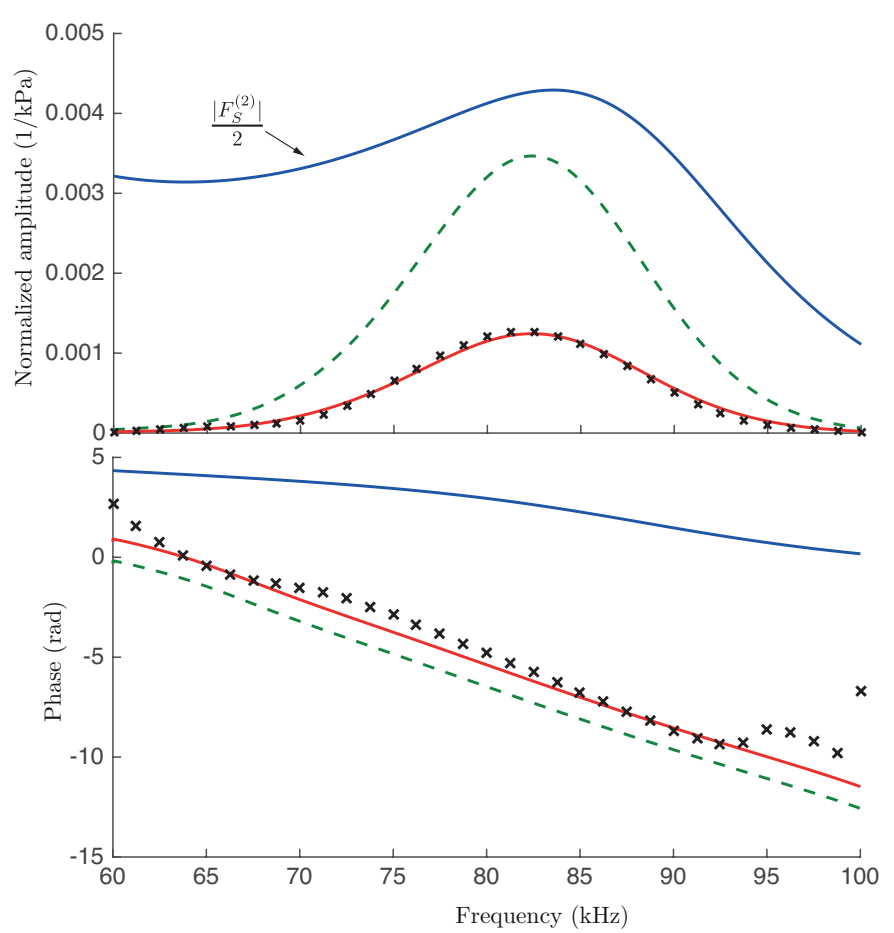

Fig. 6: Comparison between the spectrum (amplitude and phase) of the measured harmonic signal (black crosses) and three different theoretical estimates. Amplitudes were normalized by the square of the incoming signal. In blue, the nonlinear response function $F_{S}^{(2)}(f / 2)$ (see Eq. (8)). Note that for a better comparison, the amplitude of $F_{s}^{(2)}$ has been divided by a factor 2 . In green dashed lines, the broadband aspect of the experiment is taken into account (see Eq. (12)). In red, diffraction effects are also incorporated.

bubbles are correctly evaluated. The gas we used for injecting bubbles was nitrogen saturated with vapor of perfluorohexane to reduce the solubility of the bubbles. The thermal properties of the gas mixture were calculated [15]: $\gamma=1.12$ for the ratio of specific heats, and $D=2.5 \mathrm{~mm}^{2} / \mathrm{s}$ for the thermal diffusivity. With these values, the thermal damping rate [16] was estimated at $\delta_{\text {the }}=0.010$ (smaller than for bubbles of pure air). For the viscous losses [17], rheological measurements up to a frequency of $100 \mathrm{~Hz}$ were extrapolated to estimate a viscosity of $24 \mathrm{mPa} . \mathrm{s}$ at $40 \mathrm{kHz}$, giving a viscous damping rate of $\delta_{\text {vis }}=0.078$.

Nonlinear results. Fig. 6 shows the spectrum of the measured nonlinear signal $\tilde{S}_{N L}(f)$ (symbols), normalized by the integral of the incoming signal squared $\left(P / P_{R}\right)^{2} \int\left|\tilde{S}_{R}(f)\right|^{2} \mathrm{~d} f$. For a quasi monochromatic experiment, one could expect this quantity to give directly the nonlinear response function $F_{s}^{(2)}(f / 2)$ (see Eq. (7)). However, as clearly visible in Fig. 6, neither the amplitude nor the phase of the nonlinear response function are close to the measurements. There are two reasons for this discrepancy. First, the experiments are not monochromatic, which means that the broadband incoming signal contains many couples of frequencies $\left(f_{1}, f_{2}\right)$ that generate the mea-

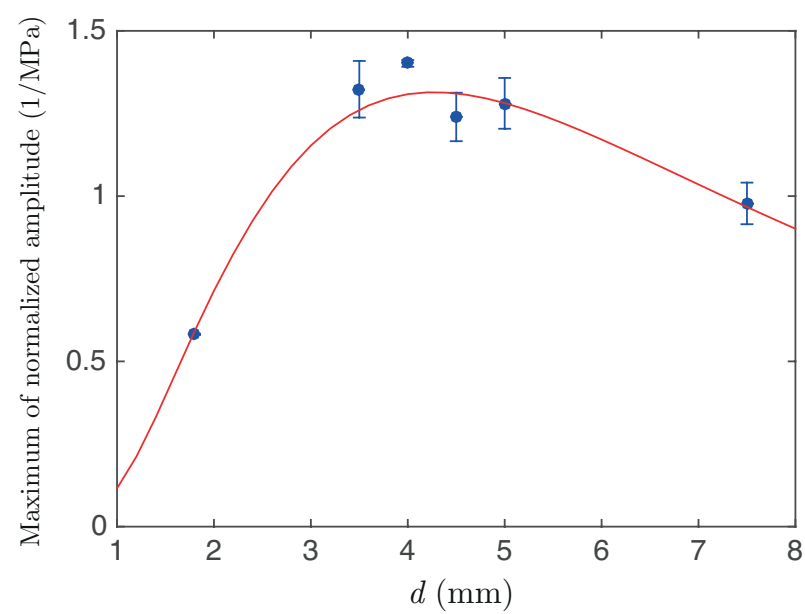

Fig. 7: Maximum of the nonlinear spectrum as a function of the distance between the bubbles, measured experimentally (symbols) and predicted by the model (red line).

sured $2 f$ signal. One should then rather consider

$$
\tilde{S}_{N L}(f)=\frac{P^{2}}{P_{R}^{2}} \int \tilde{S}_{R}(u) \tilde{S}_{R}(f-u) F_{s}^{(2)}(u, f-u) \mathrm{d} u .
$$

Destructive interferences between the different contributions make the total nonlinear response substantially weaker than $F_{s}^{(2)}(f / 2)$, as can be observed in Fig. 6 (dashed lines). With Eq. (12), the model prediction is closer to the measurements, but the amplitude is still largely overestimated. It comes from the second reason: diffraction. For the linear response, the diffraction over the $6 \mathrm{~cm}$ that separate the screen from the hydrophone is identical for the reference and the sample measurements, and thus diffraction terms disappear when considering the ratio of these two signals. In the nonlinear case, however, the harmonic signal is not diffracted with the same pattern. Knowing the shape of the beam at the screen aperture (well approximated by a $1.3 \mathrm{~cm}$-radius gaussian beam), we can use a Rayleigh-Sommerfeld integral [18] to calculate the effect of diffraction at $80 \mathrm{kHz}$. The correction mainly affects the amplitude, which is reduced by a factor 0.38 , and it yields an excellent agreement with the experimental data, as can be seen in Fig. 6. The large phase shift of the nonlinear response is responsible of the delay observed in the time domain.

Harmonic generation measurements can be repeated with bubble screens of different concentrations. Figure 7 presents the maximum of the nonlinear spectrum as a function of the lattice parameter $d$. As predicted by the model, there is an optimal concentration, around $d=4 \mathrm{~mm}$.

Conclusion. - Careful experiments with stable and well-characterized samples of bubble screens have allowed us to validate a perturbation method for calculating the harmonic generation of such structures. The interplay be- 
tween multiple scattering and nonlinearity has been clearly identified, and we showed that an optimal concentration of bubbles exists, for which the decrease of the total exciting pressure due to multiple scattering is balanced by the number of nonlinear sources.

These experimental results make bubble screens valuable candidates for new sub-wavelength nonlinear sources. Note that the theoretical maximal value of $F_{s}^{(2)}=$ $8 \mathrm{MPa}^{-1}$ (see Fig. 6) should be accessible experimentally with a monochromatic excitation and a larger sample to reduce diffraction. With such a screen, a $60 \mathrm{kPa}$ incident wave at $42 \mathrm{kHz}$ would give a nonlinear signal with a $0.008 \times 60^{2}=30 \mathrm{kPa}$ amplitude. Even better nonlinear performance can be expected if one manages to reduce the damping rate of the bubbles (see Eq. (10)), by reducing the viscosity of the medium for example. One could imagine to use such a system for the design of subwavelength acoustic "diodes" [19], blocking the acoustic energy in one direction and letting it flow in the other direction. A first screen would be optimized for being a strong nonlinear source $(f \rightarrow 2 f)$, while the second one would be optimized to block (linearly) frequency $f$ and transmit frequency $2 f$.

Further optimization of the nonlinear effects can be considered by applying our simple model to more complex screens. For instance, adding a second size of bubbles in the screen could have an interesting effect, especially if the size is chosen in such a way that they resonate at $2 f$.

We acknowledge funding support from the Agence Nationale de la Recherche (ANR-11BS09-007-03), project DiAMAN. We thank O. Bou Matar and G. Renaud for fruitful discussions.

\section{REFERENCES}

[1] Foldy L. L., Phys. Rev., 67 (1945) 107-119.

[2] Derode A., Mamou V. and Tourin A., Phys. Rev. E, 74 (2006) 036606.

[3] Wiersma D. S., Bartolini P., LagendiJk A. and Righin R., Nature, 390 (1997) 671-673.

[4] Hu H., Strybulevych A., Page J. H., Skipetrov S. E. and van Tiggelen B. A., Nature Physics, 4 (2008) 945-948

[5] McCall S. L. and Hahn E. L., Physical Review, 183 (1969) 457.

[6] Franken P., Hill A., Peters C. and Weinreich G., Phys. Rev. Lett., 7 (1961) 118.

[7] Jia X., Phys. Rev. Lett., 93 (2004) 154303.

[8] Tournat V., Zaitsev V., Gusev V., Nazarov V., Béquin P. and Castagnede B., Phys. Rev. Lett., 92 (2004) 085502.

[9] Zabolotskaya E. A. and Soluyan S. I., Sov. Phys. Acoust., 19 (1974) .

[10] Zabolotskaya E. A., Sov. Phys. Acoust., 21 (1975) .

[11] Wu J. and Tong J., Ultrasound in Med. and Biol., 24 (1997) 153-159
[12] Zaitsev V. Y., Nazarov V. E. and Belyaeva I. Y., Acoust. Phys., 47 (2001) 178-183.

[13] Plesset M. S. and Prosperetti A., Annual Review of Fluid Mechanics, 9 (1977) 145-185.

[14] Leroy V., Strybulevych A., Scanlon M. G. and Page J. H., Eur. Phys. J. E, 29 (2009) 123-130.

[15] Lindsay A. L. and Bromley L. A., Industrial and engineering chemistry, 42 (1950) .

[16] Prosperetti A., J. Acoust. Soc. Am, 61 (1977) 17-27.

[17] Devin C., J. Acoust. Soc. Am, 31 (1959) 1654-1667.

[18] Wolf E. and Marchand E. W., J. Opt. Soc. Am., 54 (1964) 587-594.

[19] Liang B., Yuan B. and Cheng J. C., Phys. Rev. Lett., 103 (2009) 104301 